\title{
Smoldering Multiple Myeloma: What Is the Threshold to Start Treatment?
}

\author{
Presented by Natalie S. Callander, MD
}

\begin{abstract}
Smoldering multiple myeloma represents approximately $10 \%$ to $15 \%$ of all myeloma cases, but more thorough evaluation may decrease that number in the future. Risk stratification is important in this patient population to avoid overtreatment or undertreatment. Patients with low-risk disease can be observed without treatment, but those at higher-risk should be enrolled on clinical trials. If a clinical trial is not an option in these patients, a time-limited intervention with lenalidomide \pm dexamethasone can be considered.
\end{abstract}

J Natl Compr Canc Netw 2020;18(12.5):1777-1779 doi: 10.6004/jnccn.2020.5043

\begin{abstract}
Although the term "smoldering myeloma" was coined in the late 1970s, ${ }^{1}$ much debate still surrounds its definition. According to Natalie S. Callander, MD, Professor of Medicine, University of Wisconsin Carbone Cancer Center, patients defined as having low-risk smoldering multiple myeloma (SMM) can forego treatment, but follow-up is necessary, she stated at the NCCN 2020 Virtual Congress: Hematologic Malignancies. However, patients with highrisk disease should be enrolled on clinical trials; in the absence of a trial option, lenalidomide-based therapy is recommended.
\end{abstract}

According to the Mayo Clinic classification system, the current definition of SMM entails the presence of monoclonal plasma cells in the bone marrow at $\geq 10 \%$ but not more than $60 \%$ and/or the presence of a monoclonal protein $\geq 3 \mathrm{~g} / \mathrm{dL}$ in the serum or $\geq 500 \mathrm{mg}$ of monoclonal protein in a 24-hour urine specimen. ${ }^{2}$ Figure 1 describes the Mayo Clinic classification of SMM and the Spanish definition, ${ }^{3}$ which focuses on phenotypically aberrant plasma cells and immunoparesis, a condition in which at least one uninvolved immunoglobulin class is reduced. The latter classification also uses a different definition of what constitutes a high protein level: in this case, a gram (rather than $500 \mathrm{mg}$ ) per 24-hour urine collection, and $2 \mathrm{~g} / \mathrm{dL}$ of IgA monoclonal protein.

An examination of 86,000 patients with MM in the National Cancer Database yielded a $14 \%$ incidence of SMM, with a higher incidence in women, African Americans, and older people (median age at diagnosis, 67 years). According to Dr. Callander, SMM generally represents approximately $10 \%$ to $15 \%$ of individuals classified as having MM. "We don't really know the true epidemiology of SMM, due to the fact that in the United States there is no [ICD-10] code separating it from MM," she noted. "So it gets lumped in with actual MM, with patients with SMM typically thought to be those who do not appear to be receiving active treatment after diagnosis."

\section{Natural History of SMM}

Patients with SMM are at an increased risk for developing MM. A 2007 study of approximately 3,500 patients confirmed this increased risk of progression: $8 \%$ of patients met the definition of SMM, and of those, 59\% ultimately developed MM. ${ }^{5}$ "Within a couple of years, a group of patients in the study had a very rapid progression to MM," Dr. Callander said. "In trying to tease out who was in this group of fast progressors, they found a couple of things: very high monoclonal protein levels $(>4 \mathrm{~g} / \mathrm{dL})$ and the presence of immunoparesis."

Researchers then found that the risk of disease progression increased to between $80 \%$ and $90 \%$ within 2 years among patients with $\geq 60 \%$ plasma cells or $\geq 100$ free light chain (involved over uninvolved) ratio ${ }^{6,7}$ Based on this research, patients with "ultra-high risk" SMM were reclassified as having MM. ${ }^{2}$ "These are 2 of the 3 factors that led to the SLiM CRAB definition," she said. "Because these patients have such a high risk for developing MM, why wait? We would just incorporate them into the definition for MM."

The SLiM CRAB criteria identifies patients who should be treated for myeloma. The "S" stands for $60 \%$ : patients with $\geq 60 \%$ clonal plasma cells in the bone marrow are likely to experience disease progression and should thus be classified as having symptomatic MM. The "Li" stands for light chains because patients with a kappa-to-lambda or lambda-to-kappa ratio of $\geq 100$. Finally the "M" stands for MRI: patients with focal bone marrow deposits of MM $>0.5 \mathrm{~cm}$ found on MRI should 

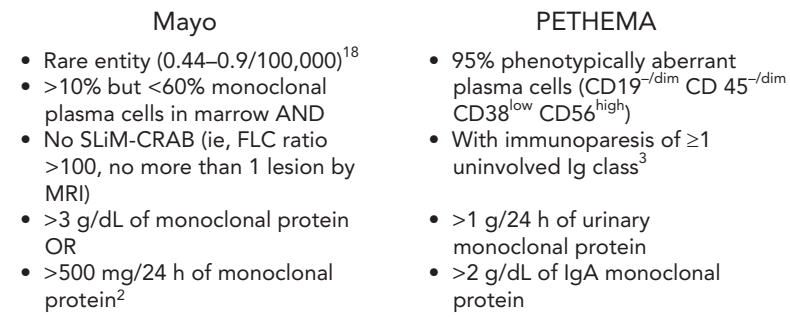

Figure 1. Current definition of smoldering multiple myeloma: much debate.

Abbreviation: FLC, free light chain.

be classified as symptomatic MM and therapy should be initiated. "So any one of those 3 features, even if a person is completely well, now constitutes $\mathrm{MM}$, in addition to the typical CRAB-defining features that we're familiar with," Dr. Callander said.

\section{Risk Stratification in SMM}

Dr. Callander noted that some type of advanced imaging modality-including whole-body MRI, pelvic/spine MRI, low-dose whole-body CT, or FDG-PET/CT-is necessary to differentiate SMM from MM (because it is much better than skeletal survey). She also noted that the imaging must be performed prior to classifying a patient as having SMM.

In a study of 120 patients with SMM who had a PET scan but did not have "myeloma-defining" results, those who had some sort of additional abnormality on PET scan (ie, slight marrow enhancement) had a 3-fold risk of progression over 3 years than those who did not have additional abnormalities. " "This is a very intriguing finding, and something that may lead to further refinement of SMM risk in the future, if this finding can be replicated," she noted.

According to Dr. Callander, the "20/20/20" model will likely be used with greater frequency to risk-stratify patients with SMM for progression. A study by Lakshman et $\mathrm{al}^{9}$ examined 421 patients with SMM and identified 3 separate clinical/biological features that seemed to be important in predicting risk of progression: $>20 \%$ plasma cells in a bone marrow biopsy, a monoclonal protein spike of $>2 \mathrm{~g} / \mathrm{dL}$, or a free light chain ratio $>20$ of involved/uninvolved. "When a patient had 0 factors, the risk of progression was 110 months on average, but if they had $\geq 2$ of these 3 features, their risk of progression to MM was 29 months," she said.

These data were recently validated in a much larger patient set. In a group of 2,004 patients with SMM with $\geq 2$ of these features, risk of progression to MM at 2 years was $46 \% .^{10}$ "So I do think that [the 20/20/20] model will probably become standard for use in other trials," she said.

Other risk determinants currently being studied include research into abnormal circulating plasma cells in blood, ${ }^{11}$ preclinical research into bacteria in the microbiome and risk of SMM progression, ${ }^{12}$ and wholegenome sequencing of specific genetic signatures that might help predict patients who will evolve. ${ }^{13}$

"One of the things that is apparent is that these different risk stratification methods don't all cover the same patients," she said. "There is not a perfect overlap, so before you hang your hat on any of these methods, you probably still need to use a bit of caution until we understand more."

\section{The Ongoing Debate}

According to Dr. Callander, the debate continues regarding whether patients with SMM should receive modest treatment to slow disease progression to active MM or whether they should undergo aggressive treatment to completely stop progression to MM.

In a recently published trial, patients with SMM were randomized to either receive lenalidomide until progression or undergo observation. ${ }^{14}$ Initially, the study focused only on patients with high-risk SMM; however, due to accrual issues, the study eventually included any patient with this disease. "The study reveals that the use of lenalidomide appeared to slow progression to MM by a hazard ratio of 0.24 , which is quite striking," she said. "So at 3 years' follow-up, patients receiving lenalidomide had only a $7.3 \%$ risk of progression versus a $31.6 \%$ risk without treatment." Although approximately half of those in the lenalidomide group experienced some grade 3 toxicity, treatment was tolerable for approximately 2 years and quality-of-life assessment did not seem to differ between the groups. Additionally, there was no apparent impact on secondary malignancies with treatment.

However, patients with low-risk SMM in the trial ${ }^{14}$ did not seem to do better or worse with either intervention, she noted. "And one very interesting finding that still remains to be explained is that the 31 Black patients on this study did not seem to benefit from the intervention," said Dr. Callander. "We don't know whether that's just because the numbers were low, but that's still under investigation."

Other SMM trials are investigating the use of myeloma-type treatments to treat SMM and potentially eradicate it. One of the first examples was a trial of 18 patients with SMM who received carfilzomib/ lenalidomide/dexamethasone (KRd) for 8 cycles, followed by 2 years of lenalidomide maintenance. ${ }^{15}$ Patients experienced high response rates, with $100 \%$ having a very good partial response or better. However, based on the current definitions of SMM, this study included a few patients with MM because some patients met the SLiM CRAB criteria for active MM.

Probably the most aggressive treatment to date comes from the GEM-CESAR trial out of Spain, she said. 
Ninety patients with SMM (per the Spanish definition, although patients did receive advanced imaging) were treated with KRd for 6 cycles, followed by an autologous stem cell transplant, followed by 2 more cycles of KRd, then lenalidomide + dexamethasone for 2 years. ${ }^{16}$ This trial also showed very high response rates, although with the caveat that again one-third of patients met the SLiM CRAB definition of MM. "But the data are very impressive. The overall survival rate was $98 \%$ at 28 months, and the progression-free survival rate was $93 \%$," she reported. "There was a death during induction that should be kept in mind, and there was some progression after relapse from complete response, so I'm still waiting to see how this all turns out." A companion US trial is currently enrolling and will use a similarly aggressive treatment (ASCENT; ClinicalTrials.gov Identifier: NCT03289299), although currently does not include transplant.

In terms of incorporating newer drugs, the CENTAURUS trial explored various dosing schedules of daratumumab in patients with SMM, and although lower response rates were observed compared with lenalidomide, patients also experienced a shorter treatment time. ${ }^{17} \mathrm{~A}$ phase III study is currently underway (ClinicalTrials.gov identifier: NCT03301220).

"In ECOG, we have tried to run the middle ground," she said. "Instead of looking at only modest treatment or very aggressive treatment, we think our patients with high-risk SMM will benefit most from reasonable time-limited, myeloma-type treatment." Dr. Callander and her colleagues are currently comparing overall survival in a phase III trial of 288 patients with high-risk SMM randomized to 2 years of daratumumab/lenalidomide/ dexamethasone or lenalidomide/dexamethasone (ClinicalTrials.gov Identifier: NCT03937635). Among other secondary endpoints, they will examine quality-of-life measurements. "By definition, these patients are asymptomatic, and we're asking them to go on chemotherapy; that is not a trivial ask," she explained. "We're also doing something very interesting here: we're asking patients and their providers to simultaneously rate what they perceive as side effects, and we think these data will be very important."

In summary, said Dr. Callander, clinical trials support the idea that patients with low-risk disease do not require treatment, but follow-up is appropriate. For patients at high-risk, enrollment on a clinical trial is preferred, and the data support lenalidomide-based therapy offprotocol, based on the 2 published randomized trials, both of which used lenalidomide. "Finally, I want to underscore that for patients on the one-arm, aggressive treatment trials, there is no comparator," she added. "So at this time, I don't think we would recommend using that kind of very aggressive myelomastyle treatment."

Disclosures: Dr. Callander has disclosed that she has no relevant financial relationships.

Correspondence: Natalie S. Callander, MD, University of Wisconsin Carbone Cancer Center, 600 Highland Avenue, Madison, WI 53792. Email: nsc@medicine.wisc.edu

\section{References}

1. Kyle R, Elveback LR. Management and prognosis of multiple myeloma. Mayo Clin Proc 1976;51:751-760.

2. Rajkumar SV, Dimopoulos MA, Palumbo A, et al. International Myeloma Working Group updated criteria for the diagnosis of multiple myeloma. Lancet Oncol 2014;15:e538-548.

3. Pérez-Persona E, Vidriales MB, Mateo G, et al. New criteria to identify risk of progression in monoclonal gammopathy of uncertain significance and smoldering multiple myeloma based on multiparameter flow cytometry analysis of bone marrow plasma cells. Blood 2007;110: 2586-2592.

4. Ravindran A, Bartley AC, Holton SJ, et al. Prevalence, incidence and survival of smoldering multiple myeloma in the United States. Blood Cancer J 2016;6:e486.

5. Kyle RA, Remstein ED, Therneau TM, et al. Clinical course and prognosis of smoldering (asymptomatic) multiple myeloma. N Engl J Med 2007;356: 2582-2590.

6. Larsen JT, Kumar SK, Dispenzieri A, et al. Serum free light chain ratio as a biomarker for high-risk smoldering multiple myeloma. Leukemia 2013;27: 941-946.

7. Waxman AJ, Mick R, Garfall AL, et al. Classifying ultra-high risk smoldering myeloma. Leukemia 2015;29:751-753.

8. Zamagni E, Nanni C, Gay F, et al. 18F-FDG PET/CT focal, but not osteolytic, lesions predict the progression of smoldering myeloma to active disease. Leukemia 2016;30:417-422.

9. Lakshman A, Rajkumar SV, Buadi FK, et al. Risk stratification of smoldering multiple myeloma incorporating revised IMWG diagnostic criteria. Blood Cancer J 2018;8:59.

10. San Miguel J, Mateos MV, Gonzalez V, et al. Updated risk stratification model for smoldering multiple myeloma (SMM) incorporating the revised
IMWG diagnostic criteria [abstract]. J Clin Oncol 2019;37(Suppl): Abstract 8000

11. Gonsalves WI, Rajkumar SV, Dispenzieri A, et al. Quantification of circulating clonal plasma cells via multiparametric flow cytometry identifies patients with smoldering multiple myeloma at high risk of progression. Leukemia 2017;31:130-135.

12. Calcinotto A, Brevi A, Chesi M, et al. Microbiota-driven interleukin-17producing cells and eosinophils synergize to accelerate multiple myeloma progression. Nat Commun 2018;9:4832.

13. Bolli N, Maura F, Minvielle $S$, et al. Genomic patterns of progression in smoldering multiple myeloma. Nat Commun 2018;9:3363.

14. Lonial S, Jacobus S, Fonseca R, et al. Randomized trial of lenalidomide versus observation in smoldering multiple myeloma. J Clin Oncol 2020; 38:1126-1137.

15. Mailankody S, Kazandjian D, Korde N, et al. Baseline mutational patterns and sustained MRD negativity in patients with high-risk smoldering myeloma. Blood Adv 2017;1:1911-1918.

16. Mateos MV, Lopez JM, Rodrigues-Otero $P$, et al. Curative strategy for high-risk smoldering myeloma (GEM-CESAR): carfilzomib, lenalidomide and dexamethasone (KRd) as induction followed by HGT-ASCT, consolidation with KRd and maintenance with Rd [abstract]. Blood 2017; 130(Suppl 1):Abstract 402.

17. Landgren CO, Chari A, Cohen YC, et al. Daratumumab monotherapy for patients with intermediate-risk or high-risk smoldering multiple myeloma: a randomized, open-label, multicenter, phase 2 study (CENTAURUS) Leukemia 2020;34:1840-1852.

18. Kristinsson SY, Holmberg E, Blimark C, et al. Treatment for high-risk smoldering myeloma. N Engl J Med 2013;369:1762-1763. 